\title{
Influence of Skeletal Muscle Volume Loss During Lenvatinib Treatment on Prognosis in Unresectable Hepatocellular Carcinoma: A Multicenter Study in Tohoku, Japan
}

\author{
Masashi Fujita ( $\nabla$ mfujita@fmu.ac.jp ) \\ Fukushima Medical University \\ Kazumichi Abe \\ Fukushima Medical University \\ Hidekatsu Kuroda \\ Iwate Medical University \\ Takayoshi Oikawa \\ Iwate Medical University \\ Masashi Ninomiya \\ Tohoku University \\ Atsushi Masamune \\ Tohoku University \\ Kazuo Okumoto \\ Yamagata University \\ Tomohiro Katsumi \\ Yamagata University \\ Wataru Sato \\ Akita University \\ Katsunori ljima \\ Akita University \\ Tetsu Endo \\ Hirosaki University \\ Shinsaku Fukuda \\ Hirosaki University \\ Nobukazu Tanabe \\ Sendai Medical Center \\ Hiroshi Mumao \\ Aomori Prefectural Central Hospital \\ Yasuhiro Takikawa \\ Iwate Medical University \\ Yoshiyuki Ueno \\ Yamagata University \\ Hiromasa Ohira \\ Fukushima Medical University
}

\section{Research Article}

Keywords: skeletal muscle volume loss, sarcopenia, hepatocellular carcinoma, lenvatinib

Posted Date: February 7th, 2022

DOI: https://doi.org/10.21203/rs.3.rs-1325658/v1 
License: @ (1) This work is licensed under a Creative Commons Attribution 4.0 International License. Read Full License

Version of Record: A version of this preprint was published at Scientific Reports on April 20th, 2022. See the published version at https://doi.org/10.1038/s41598-022-10514-3. 


\section{Abstract}

Sarcopenia is associated with poor prognosis of patients with hepatocellular carcinoma (HCC). We investigated the association of skeletal muscle volume (SMV) and its change in HCC patients taking lenvatinib. In $132 \mathrm{HCC}$ patients, psoas mass index (PMI) was calculated as the left-right sum of the vertical $\times$ horizontal diameter of psoas muscle at the third lumbar vertebra, divided by height squared. Patients were classified into two groups (low and normal PMI) based on indices of $<6.0 \mathrm{~cm}^{2} / \mathrm{m}^{2}$ for man and $<3.4 \mathrm{~cm}^{2} / \mathrm{m}^{2}$ for women. Change in PMI per month during the lenvatinib administration period $(\triangle \mathrm{PMI} / \mathrm{m})$ was calculated; and patients were classified into two groups (severe and mild atrophy) based on the $\Delta \mathrm{PMI} / \mathrm{m}$ rate, as $\geq 1 \%$ or $<1 \%$, respectively. There was no significant difference in Overall survival (OS) between the low and normal PMI groups at the start of lenvatinib administration. OS was significantly lower in the severe atrophy group than in the mild atrophy group (median; 15.2 vs. 25.6 months, $P=0.004$ ). Multivariate analysis revealed a significant association of severe atrophy with OS (hazard ratio 2.503, $P=0.007$ ). Progressive loss of SMV is a strong predictor of poor prognosis in HCC patients taking lenvatinib.

\section{Introduction}

Hepatocellular carcinoma (HCC) is the sixth most common malignancy worldwide. ${ }^{1}$ Until 2019, the clinical guidelines of the Japan Society of Hepatology (JSH) ${ }^{2}$ specified therapy with molecular targeting agents (MTAs),including sorafenib (SOR) ${ }^{3}$, regorafenib ${ }^{4}$, lenvatinib (LEN) ${ }^{5}$ and ramucirumab ${ }^{6}$, as the standard treatmentfor patients with unresectable HCC (u-HCC)and vascular invasion or extrahepatic metastasis.In 2020, immune checkpoint inhibitor (ICI) therapy including the combination of atezolizumab and bevacizumab ${ }^{7}$ was approved in Japanas the first-line treatment for unresectable HCC. ${ }^{8}$ However, MTAs are still used as the second-line treatment and remain important because of the convenience of the administration method.

Skeletal muscle volume (SMV) loss is animportantfactorof sarcopenia that is associated with liver disease. Hanai et al reported that SMV decreased by $2.2 \%$ per year in patients with liver cirrhosis, and the rate of decrease increased in parallel with the severity of cirrhosis. ${ }^{9}$ Sarcopenia has a negative impact on mortality in patients withchronic liver disease (CLD) ${ }^{10}$ and u-HCC treated with SOR ${ }^{11,12}$ and LEN. ${ }^{13,14}$ Several studies have reported that decline of SMV during the treatment period is a criticalfactor in patients with HCC treated with SOR or LEN. ${ }^{12,15}$ However, these reports estimated change in SMVonly over a short period from the start of LEN.

We have previously reported that change in the skeletal muscle index (SMI) was a risk factor in the patients with HCC patients treated with transarterial chemoembolization. ${ }^{16}$ In that study, we used the psoas muscle index (PMI) as a simpler method for determining SMI, calculated as the vertical $\times$ horizontal diameter of the psoas muscle at the third lumbar vertebra (L3) on computed tomography (CT), divided by height squared. ${ }^{17}$

The present study aimed to evaluate the long-term effects of SMVloss in patients with u-HCCby examining changes in PMI at the start of LEN administration (PMI-Pre), at the time of the first judgement of therapeutic effect (PMI-1st), and at the end of LEN administration (PMI-Post).

\section{Results}

\section{Baseline characteristics}

Table 1 shows the baseline (Pre) characteristics of patients. Median age of the 132 patients was 70 years (range, 38-89 years) and 109 patients (82.6\%) were men. The median observation period after the start of LEN administration was 11.0 months (range, 1.9-32.7 months). The median HCC treatment period before administration of LEN was 14.8 months (range, 0-132.8 months). The causative disease of HCC was hepatitis B virus (HBV; $n=28)$, hepatitis $C$ virus $(\mathrm{HCV} ; n=35)$, alcohol $(n=38)$, non-alcoholic fatty liver disease (NAFLD; $n=27)$, and other $(n=4)$. Fourteen patients $(10.6 \%)$ had moderate loss of liver function reserve (Child-Pugh class $B)$. The median albumin-bilirubin (ALBI) score was -2.40 and 51 patients (38.6\%) had loss of liver function reserve (modified ALBI [mALBI] grade $2 \mathrm{~b}$ or 3$)$. Forty-nine patients $(37.1 \%)$ had macrovascular invasion (MVI) and 37 patients $(28.0 \%)$ had extrahepatic metastasis. Extrahepatic metastasis was to lymph nodes $(n=12)$, lung $(n=19$, bone $(n=7)$, adrenal glands $(n=7)$, and carcinomatous peritonitis $(n=6)$. One-hundred-thirteen patients $(85.6 \%)$ had received other treatment prior to administration of LEN. The breakdown of treatment was as follows: surgery $(n=26)$, local treatment $(n=13)$, hepatic intra-arterial therapy $(n=70)$, radiation therapy $(n=2)$, and other 
treatment $(\mathrm{n}=2)$. The median administration period was 7.7 months (range, $0.3-32.7$ months). Ninety-seven patients $(73.5 \%)$ discontinued LEN, most commonly due to progressive disease (PD) $(n=44)$. Fifty-two patients $(39.4 \%)$ died during the follow-up period. 


\begin{tabular}{|c|c|}
\hline Variable & Total $(n=132)$ \\
\hline Observation period after initiation of LEN (months) & $11.0(7.7-17.3)$ \\
\hline Sex (male/female) & $109 / 23$ \\
\hline Age (years) & $70(65-76)$ \\
\hline $\mathrm{BMI}\left(\mathrm{kg} / \mathrm{m}^{2}\right)$ & $23.5(21.2-25.5)$ \\
\hline Etiology (HBV/HCV/alcohol/NAFLD/other) & $28 / 35 / 38 / 27 / 4$ \\
\hline Child-Pugh class (A/B) & $118 / 14$ \\
\hline mALBI grade $(1 / 2 a / 2 b / 3)$ & $50 / 31 / 47 / 4$ \\
\hline TNM stage (II/III/IVA/IVB) & $21 / 48 / 26 / 37$ \\
\hline Maximum tumor diameter (cm) & $4(2.5-7.5)$ \\
\hline Number of tumors & $4(2-10)$ \\
\hline Up-to-7 criteria (in/out/no liver tumor) & $50 / 70 / 12$ \\
\hline Macrovascular invasion (yes/no/no liver tumor) & $49 / 71 / 12$ \\
\hline Metastasis (yes/no) & $37 / 95$ \\
\hline HCC (recurrence/naive) & $113 / 19$ \\
\hline Total bilirubin (mg/dL) & $0.8(0.7-1.0)$ \\
\hline Albumin (g/dL) & $3.7(3.4-4.1)$ \\
\hline Prothrombin time-international normalized ratio & $1.05(0.97-1.13)$ \\
\hline Platelet count $\left(\cdot 10^{4} / \mu \mathrm{L}\right)$ & $14.8(10.2-18.6)$ \\
\hline Choline-esterase (U/L) † & $199(153-261)$ \\
\hline Total cholesterol $(\mathrm{mg} / \mathrm{dL}) \dagger$ & $165(147-180)$ \\
\hline Triglyceride $(\mathrm{mg} / \mathrm{dL}) \dagger$ & $98(76-130)$ \\
\hline LDL-C (mg/dL) † & $89(69-115)$ \\
\hline Hemoglobin A1c (\%) † & $5.9(5.5-6.5)$ \\
\hline Ammonia $(\mu \mathrm{g} / \mathrm{dL}) \dagger$ & $48(37-63)$ \\
\hline $\operatorname{AFP}(\mathrm{ng} / \mathrm{dL})$ & $43(7.2-654)$ \\
\hline PIVKA-II (mAU/mL) & $472(50-2064)$ \\
\hline Initial dose of LEN (4/8/12 mg) & $8 / 77 / 47$ \\
\hline Dose down (yes/no/unknown) & $64 / 64 / 4$ \\
\hline Administration period of LEN (months) & $7.7(3.1-12.2)$ \\
\hline Discontinued LEN (yes/no) & $97 / 35$ \\
\hline
\end{tabular}

Values are presented as the median (interquartile range). LEN: lenvatinib, BMI: body mass index, HBV: hepatitis B virus, HCV: hepatitis C virus, NAFLD: non-alcoholic fatty liver disease, mALBI: modified albumin-bilirubin, TNM: tumor node metastasis, HCC: hepatocellular carcinoma, LCL-C: low density lipoprotein cholesterol, AFP: alpha fetoprotein, PIVKA-II: protein induced by Vitamin K absence or antagonists-II, PD: progressive disease, mRECIST: Modified Response Evaluation Criteria in Solid Tumors, CR: complete response, PR: partial response, SD: stable disease, PMI: psoas muscle index, $\triangle \mathrm{PMI} / \mathrm{m}$ : change in PMI per month, $\Delta \mathrm{PMI} / \mathrm{m}$ rate: rate of change in PMI per month during administration of LEN.

† Calculated using the available data. 


\begin{tabular}{|c|c|}
\hline Variable & Total $(n=132)$ \\
\hline Reason for discontinuing LEN (adverse event/PD/other) & $39 / 44 / 14$ \\
\hline 1st mRECIST assessment (CR/PR/SD/PD/no assessment) & $0 / 46 / 39 / 42 / 5$ \\
\hline Received other treatments after discontinuing LEN (yes/no) & $59 / 38$ \\
\hline $\mathrm{PMI}\left(\mathrm{cm}^{2} / \mathrm{m}^{2}\right)$ & $5.63(4.63-6.89)$ \\
\hline$\Delta \mathrm{PMI} / \mathrm{m}\left(\mathrm{cm}^{2} / \mathrm{m}^{2}\right)$ & $0.05(-0.01-0.19)$ \\
\hline$\Delta \mathrm{PMI} / \mathrm{m}$ rate $(\%)$ & $0.89(-0.19-3.75)$ \\
\hline Death (yes/no) & $52 / 80$ \\
\hline \multicolumn{2}{|c|}{$\begin{array}{l}\text { Values are presented as the median (interquartile range). LEN: lenvatinib, BMI: body mass index, HBV: hepatitis B virus, HCV: } \\
\text { hepatitis C virus, NAFLD: non-alcoholic fatty liver disease, mALBI: modified albumin-bilirubin, TNM: tumor node metastasis, HCC: } \\
\text { hepatocellular carcinoma, LCL-C: low density lipoprotein cholesterol, AFP: alpha fetoprotein, PIVKA-Il: protein induced by Vitamin K } \\
\text { absence or antagonists-II, PD: progressive disease, mRECIST: Modified Response Evaluation Criteria in Solid Tumors, CR: complete } \\
\text { response, PR: partial response, SD: stable disease, PMl: psoas muscle index, } \triangle \mathrm{PMI/m:} \mathrm{change} \mathrm{in} \mathrm{PMI} \mathrm{per} \mathrm{month,} \triangle \mathrm{PMI} / \mathrm{m} \text { rate: rate } \\
\text { of change in PMI per month during administration of LEN. }\end{array}$} \\
\hline † Calculated using the available data. & \\
\hline
\end{tabular}

The median PMI-Pre by etiology was $5.84 \mathrm{~cm}^{2} / \mathrm{m}^{2}$ for HBV, $5.52 \mathrm{~cm}^{2} / \mathrm{m}^{2}$ for HCV, $6.20 \mathrm{~cm}^{2} / \mathrm{m}^{2}$ for alcohol, $5.37 \mathrm{~cm}^{2} / \mathrm{m}^{2}$ for NAFLD, and $5.16 \mathrm{~cm}^{2} / \mathrm{m}^{2}$ for other. The median change in PMI per month $(\triangle \mathrm{PMI} / \mathrm{m})$ rate $\left(\triangle \mathrm{PMI}{ }^{\star} 100 / \mathrm{PMI}\right.$-Pre) by etiology was $0.55 \%$ for $\mathrm{HBV}$, $0.56 \%$ for $\mathrm{HCV}, 1.32 \%$ for alcohol, $2.47 \%$ for NAFLD, and $1.87 \%$ for other. There was no significant difference in PMI-Pre or $\triangle \mathrm{PMI} / \mathrm{m}$ rate in terms of etiology $(P=0.27$ and $P=0.106$, respectively).

\section{Change in PMI during the LEN administration period}

For all patients, median PMI-Pre, PMI-1st, and PMI-Post were 5.63, 5.62, and $4.97 \mathrm{~cm}^{2} / \mathrm{m}^{2}$, respectively. A significant difference was found between each of PMI-Pre and PMI-1st $(P=0.011)$, PMI-Pre and PMI-Post $(P<0.001)$, and PMI-1st and PMI-Post $(P<0.001)$

(Fig. 1). Median change in PMI per month during the LEN administration period $\left(\triangle \mathrm{PMI} / \mathrm{m}\right.$ ) was $0.05 \mathrm{~cm}^{2} / \mathrm{m}^{2}$ (interquartile range [IQR], 0.01 to 0.19 ). And median rate of decrease of PMI per month ( $\triangle \mathrm{PMI} / \mathrm{m}$ rate) was $0.89 \%$ (IQR, -0.19 to 3.75 ), respectively (Table 1 ).

\section{Comparison between the low and normal PMI groups at baseline}

Of the 132 patients, 64 (48.5\%) were classified as having low PMI. There was no significant difference between the low and normal PMI groups in terms of age, liver function reserve (median ALBI score, -2.43 vs. $-2.35 ; P=0.918$ ), stage of HCC including MVI and extrahepatic metastasis, or treatment (the presence, absence, or breakdown of other treatments received before and after LEN treatment). Significantly more men had low PMI than normal PMI $(P=0.005)$ (Table 2). Fewer patients in the low PMI group (PMI $<6.0$ $\mathrm{cm}^{2} / \mathrm{m}^{2}$ for men and $<3.4 \mathrm{~cm}^{2} / \mathrm{m}^{2}$ for women) received other treatments after discontinuing LEN compared with those in the normal PMl group (PMI $\geq 6.0 \mathrm{~cm}^{2} / \mathrm{m}^{2}$ for men and $\geq 3.4 \mathrm{~cm}^{2} / \mathrm{m}^{2}$ for women) $(P=0.006)$. There was no significant difference in $\Delta \mathrm{PMI} / \mathrm{m}(P=$ $0.181)$ or in $\Delta \mathrm{PMI} / \mathrm{m}$ rate $(P=0.485)$ between the low and normal PMI groups. 
Table 2

Comparison between the low and normal PMI groups

\begin{tabular}{|c|c|c|c|}
\hline Variable & Normal PMI $(n=68)$ & Low PMI $(n=64)$ & P-value \\
\hline Observation period after initiation of LEN (months) & $12.4(8.5-17.5)$ & $9.8(7.2-17.0)$ & 0.118 \\
\hline Sex (male/female) & $50 / 18$ & $59 / 5$ & 0.005 \\
\hline Age (years) & $70(65-76)$ & $70(65-76)$ & 0.624 \\
\hline $\mathrm{BMI}\left(\mathrm{kg} / \mathrm{m}^{2}\right)$ & $23.9(22.1-25.9)$ & $23.1(20.6-25.2)$ & 0.083 \\
\hline Child-Pugh class (A/B) & $62 / 6$ & $56 / 8$ & 0.497 \\
\hline mALBI grade $(1 / 2 a / 2 b / 3)$ & $27 / 16 / 24 / 1$ & $23 / 15 / 23 / 3$ & 0.541 \\
\hline TNM stage (I/II/III/IVA/IVB) & $13 / 20 / 14 / 21$ & $8 / 28 / 12 / 16$ & 0.706 \\
\hline Up-to-7 criteria (in/out/no liver tumor) & $24 / 36 / 8$ & $26 / 34 / 4$ & 0.529 \\
\hline Macrovascular invasion (yes/no/no liver tumor) & $27 / 33 / 8$ & $22 / 38 / 4$ & 0.396 \\
\hline Metastasis (yes/no) & $21 / 47$ & $16 / 48$ & 0.456 \\
\hline HCC (recurrence/naive) & $58 / 10$ & $55 / 9$ & 0.919 \\
\hline Total bilirubin $(\mathrm{mg} / \mathrm{dL})$ & $0.9(0.7-1.0)$ & $0.8(0.7-1.0)$ & 0.719 \\
\hline Albumin $(\mathrm{g} / \mathrm{dL})$ & $3.7(3.4-4.0)$ & $3.7(3.4-4.1)$ & 0.862 \\
\hline Prothrombin time-international normalized ratio & $1.05(0.98-1.13)$ & $1.04(0.96-1.13)$ & 0.389 \\
\hline Platelet count $\left(\times 10^{4} / \mu \mathrm{L}\right)$ & $14.3(10.0-17.0)$ & $15.3(12.1-20.3)$ & 0.099 \\
\hline Choline-esterase $(\mathrm{U} / \mathrm{L}) \dagger$ & $193(156-247)$ & $211(137-272)$ & 0.466 \\
\hline Total cholesterol $(\mathrm{mg} / \mathrm{dL})+$ & $171(155-184)$ & $163(144-180)$ & 0.138 \\
\hline Triglyceride $(\mathrm{mg} / \mathrm{dL}) \dagger$ & $104(79-138)$ & $93(66-114)$ & 0.101 \\
\hline LDL-C $(\mathrm{mg} / \mathrm{dL}) \dagger$ & $89(72-117)$ & $89(62-113)$ & 0.292 \\
\hline Hemoglobin A1c (\%) † & $5.9(5.4-6.7)$ & $5.9(5.5-6.4)$ & 0.621 \\
\hline Ammonia $(\mu \mathrm{g} / \mathrm{dL}) \dagger$ & $48(37-63)$ & $47(39-65)$ & 0.854 \\
\hline $\operatorname{AFP}(\mathrm{ng} / \mathrm{dL})$ & $95.6(8.3-1594)$ & $23.8(6.1-328)$ & 0.103 \\
\hline PIVKA-II (mAU/mL) & $459(34-2086)$ & $483(61-2030)$ & 0.952 \\
\hline Initial dose of LEN (4/8/12 mg) & $3 / 37 / 28$ & $5 / 40 / 19$ & 0.14 \\
\hline Dose down (yes/no/unknown) & $35 / 31 / 2$ & $29 / 33 / 2$ & 0.483 \\
\hline Administration period of LEN (months) & $8.2(3.3-12.8)$ & $6.4(2.6-11.9)$ & 0.196 \\
\hline Discontinued LEN (yes/no) & $45 / 23$ & $52 / 12$ & 0.051 \\
\hline Reason for discontinuing LEN (adverse event/PD/other) & $16 / 20 / 9$ & $23 / 24 / 5$ & 0.39 \\
\hline 1st mRECIST assessment (CR/PR/SD/PD/no assessment) & $0 / 28 / 21 / 17 / 2$ & $0 / 18 / 18 / 25 / 3$ & 0.059 \\
\hline
\end{tabular}

Values are presented as the median (interquartile range). LEN: lenvatinib, BMI: body mass index, mALBI: modified albumin-bilirubin, TNM: tumor node metastasis, HCC: hepatocellular carcinoma, LCL-C: low density lipoprotein cholesterol, AFP: alpha fetoprotein, PIVKA-II: protein induced by Vitamin K absence or antagonists-II, PD: progressive disease, mRECIST: Modified Response Evaluation Criteria in Solid Tumors, CR: complete response, PR: partial response, SD: stable disease, PMI: psoas muscle index, $\triangle \mathrm{PMI} / \mathrm{m}$ : change in PMI per month, $\triangle \mathrm{PMI} / \mathrm{m}$ rate: rate of change in PMI per month during administration of LEN.

Low PMl group: PMI $<6.0 \mathrm{~cm}^{2} / \mathrm{m}^{2}$ for men and $<3.4 \mathrm{~cm}^{2} / \mathrm{m}^{2}$ for women, normal PMl group: $\mathrm{PMI} \geq 6.0 \mathrm{~cm}^{2} / \mathrm{m}^{2}$ for men and $\geq 3.4$ $\mathrm{cm}^{2} / \mathrm{m}^{2}$ for women.

† Calculated using the available data. 


\begin{tabular}{|c|c|c|c|}
\hline Variable & Normal PMI $(n=68)$ & Low PMI $(n=64)$ & P-value \\
\hline Received other treatments after discontinuing LEN (yes/no) & $34 / 11$ & $25 / 27$ & 0.006 \\
\hline$\Delta \mathrm{PMI} / \mathrm{m}\left(\mathrm{cm}^{2} / \mathrm{m}^{2}\right)$ & $0.07(-0.01-0.26)$ & $0.04(-0.03-0.16)$ & 0.181 \\
\hline$\Delta \mathrm{PMI} / \mathrm{m}$ rate $(\%)$ & $0.97(0.07-4.03)$ & $0.89(-0.65-3.45)$ & 0.485 \\
\hline Death (yes/no) & $24 / 44$ & $28 / 36$ & 0.324 \\
\hline \multicolumn{4}{|c|}{$\begin{array}{l}\text { Values are presented as the median (interquartile range). LEN: lenvatinib, BMI: body mass index, mALBI: modified albumin-bilirubin, } \\
\text { TNM: tumor node metastasis, HCC: hepatocellular carcinoma, LCL-C: low density lipoprotein cholesterol, AFP: alpha fetoprotein, } \\
\text { PIVKA-Il: protein induced by Vitamin K absence or antagonists-II, PD: progressive disease, mRECIST: Modified Response Evaluation } \\
\text { Criteria in Solid Tumors, CR: complete response, PR: partial response, SD: stable disease, PMl: psoas muscle index, } \triangle \text { PMI/m: } \\
\text { change in PMI per month, } \triangle \text { PMI/m rate: rate of change in PMI per month during administration of LEN. }\end{array}$} \\
\hline \multicolumn{4}{|c|}{$\begin{array}{l}\text { Low PMI group: } \mathrm{PMI}<6.0 \mathrm{~cm}^{2} / \mathrm{m}^{2} \text { for men and }<3.4 \mathrm{~cm}^{2} / \mathrm{m}^{2} \text { for women, normal PMI group: } \mathrm{PMI} \geq 6.0 \mathrm{~cm}^{2} / \mathrm{m}^{2} \text { for men and } \geq 3.4 \\
\mathrm{~cm}^{2} / \mathrm{m}^{2} \text { for women. }\end{array}$} \\
\hline
\end{tabular}

\section{Assessment of prognosis}

For all patients, median survival time (MST) was 21.8 months, and the survival rate at 12 and 24 months was 70.6 and 46.9 months, respectively. There was no significant difference in MST between the low and normal PMI groups (18.1 month vs. 22.0 months, $P=$ 0.165) (Fig. 2A).

Univariate analysis revealed the following as significantly associated with overall survival (OS, months): mALBI grade $2 \mathrm{~b}$ or 3 , tumor node metastasis (TNM) stage, alpha fetoprotein (AFP) $\geq 43 \mathrm{ng} / \mathrm{dL}$, protein induced by Vitamin K absence or antagonists-II (PIVKA-II) $\geq 472 \mathrm{mAU} / \mathrm{mL}$, receiving other treatments after discontinuing $\mathrm{LEN}$, and severe muscle atrophy $(\triangle \mathrm{PMI} / \mathrm{m} \geq 1 \%)($ Table 3$)$. Multivariate analysis indicated the following as significantly associated with OS: severe muscle atrophy (hazard ratio [HR], 5.824; $95 \%$ confidence interval [Cl], 3.254-10.420; $P<0.001)$, mALBI grade $2 \mathrm{~b}$ or $3(\mathrm{HR}, 2.709 ; 95 \% \mathrm{Cl}, 1.452-5.054 ; P=0.002), \mathrm{AFP} \geq 43 \mathrm{ng} / \mathrm{dL}$ (HR, 2.687 ; $95 \% \mathrm{Cl}, 1.400-5.157 ; P=0.003)$, and PIVKA-II $\geq 472 \mathrm{ng} / \mathrm{dL}(\mathrm{HR}, 2.383 ; 95 \% \mathrm{Cl}, 1.207-4.702 ; \mathrm{P}=0.012)$. Prognosis was significantly worse in the severe atrophy group $(\mathrm{n}=65)$ than in the mild atrophy group $(\triangle \mathrm{PMl} / \mathrm{m}<1 \%)(\mathrm{MST}: 15.2$ months vs. 25.6 months, $P=$ 0.004) (Fig. 2B). Survival rates at 12 and 24 months in the severe and mild atrophy groups were $56.1 \% / 31.8 \%$ and $83.4 \% / 59.5 \%$, respectively. 
Table 3

Predictive factors for overall survival

\begin{tabular}{|c|c|c|c|c|}
\hline \multirow[t]{2}{*}{ Variable } & \multicolumn{2}{|l|}{ Univariate Analysis } & \multicolumn{2}{|l|}{ Multivariate Analysis } \\
\hline & $\mathrm{HR}(95 \% \mathrm{Cl})$ & $\begin{array}{l}P \\
\text { value }\end{array}$ & $\mathrm{HR}(95 \% \mathrm{Cl})$ & P value \\
\hline Sex (male vs. female) & $\begin{array}{l}0.746(0.360- \\
1.546)\end{array}$ & 0.43 & $1.037(0.452-2.378)$ & 0.931 \\
\hline Age (years, $\geq 70$ vs. $<70$ ) & $\begin{array}{l}0.880(0.508- \\
1.524)\end{array}$ & 0.649 & $1.020(0.548-1.897)$ & 0.951 \\
\hline mALBI grade (2b/3 vs. $1 / 2 a)$ & $\begin{array}{l}3.081(1.742- \\
5.449)\end{array}$ & $<0.001$ & $2.709(1.452-5.054)$ & 0.002 \\
\hline TNM stage (IVA/IVB vs. II/III) & $2.102(1.197-3.69)$ & 0.01 & $1.434(0.775-2.653)$ & 0.251 \\
\hline AFP (ng/dL, $\geq 43$ vs. $<43$ ) & $3.293(1.78-6.092)$ & $<0.001$ & $2.687(1.400-5.157)$ & 0.003 \\
\hline PIVKA-II (mAU/mL, $\geq 472$ vs. <472) & $2.556(1.415-4.617)$ & 0.001 & $2.383(1.207-4.702)$ & 0.012 \\
\hline $\begin{array}{l}\text { Received other treatments after discontinuing LEN (yes vs. } \\
\text { no) }\end{array}$ & $\begin{array}{l}0.456(0.251- \\
0.827)\end{array}$ & 0.01 & $0.673(0.359-1.259)$ & 0.215 \\
\hline Low PMI vs. normal PMI & $\begin{array}{l}1.469(0.851- \\
2.537)\end{array}$ & 0.168 & & \\
\hline Severe atrophy group vs. mild atrophy group & $\begin{array}{l}2.243(1.283- \\
3.919)\end{array}$ & 0.005 & $5.824(3.254-10.420)$ & $<0.001$ \\
\hline \multicolumn{5}{|c|}{$\begin{array}{l}\text { HR: hazard ratio, Cl: confidence interval, mALBI: modified albumin-bilirubin, TNM: tumor node metastasis, AFP: alpha fetoprotein, } \\
\text { PIVKA-II: protein induced by Vitamin K absence or antagonists-II, LEN: Lenvatinib, PMI: psoas muscle index. }\end{array}$} \\
\hline \multicolumn{5}{|c|}{$\begin{array}{l}\text { Low PMI group: PMI }<6.0 \mathrm{~cm}^{2} / \mathrm{m}^{2} \text { for men and }<3.4 \mathrm{~cm}^{2} / \mathrm{m}^{2} \text { for women, Normal PMI group: PMI } \geq 6.0 \mathrm{~cm}^{2} / \mathrm{m}^{2} \text { for men and } \geq 3.4 \\
\mathrm{~cm}^{2} / \mathrm{m}^{2} \text { for women. }\end{array}$} \\
\hline \multicolumn{5}{|c|}{ Severe atrophy group: $\Delta \mathrm{PMI} / \mathrm{m}$ rate $\geq 1 \%$, Mild atrophy group: $\Delta \mathrm{PMI} / \mathrm{m}$ rate $<1 \%$. } \\
\hline$(\triangle \mathrm{PMI} / \mathrm{m}$ rate: rate of change in psoas muscle index per $\mathrm{mc}$ & uring administration & LEN) & & \\
\hline
\end{tabular}

\section{Characteristics of the severe and mild atrophy groups during the administration period}

Table 4 shows the comparison of characteristics between the mild and severe atrophy groups during the administration period. The observation period was significantly shorter in the severe atrophy group than in the mild atrophy group (median; 8.7 months vs. 13.8 months, $P<0.001)$. The Pre values of body mass index (BMl, $P=0.034)$ and choline-esterase $(\mathrm{ChE} ; P=0.014)$ were lower in the severe atrophy group than in the mild atrophy group. In contrast, the Post values of liver function reserve including Child-Pugh and ALBI score, albumin, and ChE were significantly worse in the severe atrophy group than in the mild atrophy group. 
Table 4

Comparison between the severe and mild atrophy groups

\begin{tabular}{|c|c|c|c|c|c|c|}
\hline \multirow[t]{2}{*}{ Variable } & \multicolumn{3}{|c|}{ Time of start of taking LEN } & \multicolumn{3}{|c|}{ Time of end of taking LEN } \\
\hline & $\begin{array}{l}\text { Mild atrophy } \\
(n=67)\end{array}$ & $\begin{array}{l}\text { Severe atrophy } \\
(n=65)\end{array}$ & $\begin{array}{l}P \\
\text { value }\end{array}$ & Mild atrophy & Severe atrophy & $\begin{array}{l}\mathrm{P} \\
\text { value }\end{array}$ \\
\hline $\begin{array}{l}\text { Observation period after } \\
\text { initiation of LEN (months) }\end{array}$ & $13.8(9.1-19.1)$ & $8.7(6.0-14.8)$ & $<0.001$ & & & \\
\hline Sex (male/female) & $56 / 11$ & $53 / 12$ & 0.761 & & & \\
\hline Age (years) & $70(66-76)$ & $70(64-75)$ & 0.655 & & & \\
\hline BMI $\left(\mathrm{kg} / \mathrm{m}^{2}\right)$ & $23.9(22.1-25.9)$ & $22.6(20.8-25.1)$ & 0.034 & & & \\
\hline $\begin{array}{l}\text { Child-Pugh score } \\
\text { (5/6/7/8/9/10-/unknown) }\end{array}$ & $44 / 18 / 4 / 1 / 0 / 0 / 0$ & $37 / 19 / 6 / 2 / 1 / 0 / 0$ & 0.232 & $19 / 11 / 8 / 6 / 0 / 0 / 23$ & $8 / 17 / 7 / 9 / 2 / 4 / 18$ & 0.007 \\
\hline $\begin{array}{l}\text { Child-Pugh class } \\
\text { (A/B/C/unknown) }\end{array}$ & $62 / 5 / 0 / 0$ & $56 / 9 / 0 / 0$ & 0.237 & $30 / 14 / 0 / 23$ & $25 / 18 / 4 / 18$ & 0.091 \\
\hline ALBI score & $\begin{array}{l}-2.48(-2.80 \text { to } \\
-2.14)\end{array}$ & $\begin{array}{l}-2.31(-2.65 \text { to } \\
-2.08)\end{array}$ & 0.08 & $\begin{array}{l}-2.31 \\
1.96)\end{array}$ & $\begin{array}{l}-2.05(-2.39 \text { to } \\
-1.59)\end{array}$ & 0.025 \\
\hline $\begin{array}{l}\text { mALBI grade } \\
(1 / 2 a / 2 b / 3 / \text { unknown })\end{array}$ & $31 / 13 / 22 / 1 / 0$ & $19 / 18 / 25 / 3 / 0$ & 0.077 & $10 / 10 / 19 / 5 / 23$ & $5 / 8 / 26 / 8 / 18$ & 0.074 \\
\hline TNM Stage (II/III/IVA/IVB) & $10 / 27 / 10 / 20$ & $11 / 21 / 16 / 17$ & 0.956 & & & \\
\hline HCC (recurrence/naive) & $59 / 8$ & $54 / 11$ & 0.419 & & & \\
\hline Total bilirubin (mg/dL) & $0.8(0.6-1.0)$ & $0.8(0.7-1.0)$ & 0.391 & $0.9(0.7-1.3)$ & $1.1(0.7-1.9)$ & 0.322 \\
\hline Albumin (g/dL) & $3.8(3.5-4.1)$ & $3.6(3.4-3.9)$ & 0.077 & $3.6(3.3-3.8)$ & $3.4(2.9-3.7)$ & 0.018 \\
\hline $\begin{array}{l}\text { Prothrombin time- } \\
\text { international normalized } \\
\text { ratio }\end{array}$ & $1.04(0.96-1.15)$ & $1.05(0.98-1.12)$ & 0.916 & $1.02(0.95-1.12)$ & $1.02(0.98-1.20)$ & 0.586 \\
\hline Platelet count $\left(\times 10^{4} / \mu \mathrm{L}\right)$ & $14.4(10.1-18.0)$ & $15.0(10.8-18.9)$ & 0.667 & $14.0(8.9-17.6)$ & $13.1(10.2-18.8)$ & 0.46 \\
\hline Cholineesterase (U/L) † & $219(172-266)$ & $173(138-244)$ & 0.014 & $193(146-267)$ & $150(107-197)$ & 0.001 \\
\hline $\begin{array}{l}\text { Total cholesterol }(\mathrm{mg} / \mathrm{dL}) \\
+\end{array}$ & 170 (149-188) & $162(149-177)$ & 0.386 & $168(130-210)$ & $158(121-204)$ & 0.378 \\
\hline Triglyceride $(\mathrm{mg} / \mathrm{dL}) \dagger$ & $89(70-129)$ & $104(77-135)$ & 0.202 & $99(63-148)$ & $82(68-108)$ & 0.527 \\
\hline LDL-L (mg/dL) † & $85(65-112)$ & $93(70-115)$ & 0.461 & $97(71-131)$ & $92(63-115)$ & 0.356 \\
\hline Hemoglobin A1c (\%) † & $5.8(5.5-6.5)$ & $5.9(5.5-6.5)$ & 0.9 & $5.6(5.3-6.1)$ & $5.3(5.1-6.1)$ & 0.322 \\
\hline Ammonia $(\mu \mathrm{g} / \mathrm{dL}) \dagger$ & $46(34-57)$ & $53(41-66)$ & 0.052 & $45(30-52)$ & $47(34-66)$ & 0.164 \\
\hline AFP (ng/dL) & $24.3(5.7-411)$ & $97.2(8.5-2156)$ & 0.141 & $58.6(4.6-1396)$ & $59.8(6.5-1875)$ & 0.43 \\
\hline PIVKA-II (mAU/mL) & $380(34-1702)$ & $663(80.3-2136)$ & 0.271 & $\begin{array}{l}1619(262- \\
15372)\end{array}$ & $\begin{array}{l}2251(210- \\
16902)\end{array}$ & 0.563 \\
\hline
\end{tabular}

Values are presented as median (interquartile range). LEN: lenvatinib, BMI: body mass index, ALBI: albumin-bilirubin, mALBI: modified albumin-bilirubin, TNM: tumor node metastasis, HCC: hepatocellular carcinoma, LCL-C: low density lipoprotein cholesterol, AFP: alpha fetoprotein, PIVKA-II: protein induced by Vitamin K absence or antagonists-II, PD: progressive disease, mRECIST: Modified Response Evaluation Criteria in Solid Tumors, CR: complete response, PR: partial response, SD: stable disease, PMI: psoas muscle index.

Severe atrophy group: $\Delta \mathrm{PMI} / \mathrm{m}$ rate $\geq 1 \%$, Mild atrophy group: $\Delta \mathrm{PMI} / \mathrm{m}$ rate $<1 \%$.

( $\triangle \mathrm{PMI} / \mathrm{m}$ rate: rate of change in PMI per month during administration of LEN)

+ Calculated using the available data. 


\begin{tabular}{|c|c|c|c|c|c|c|}
\hline \multirow[t]{2}{*}{ Variable } & \multicolumn{3}{|c|}{ Time of start of taking LEN } & \multicolumn{3}{|c|}{ Time of end of taking LEN } \\
\hline & $\begin{array}{l}\text { Mild atrophy } \\
(n=67)\end{array}$ & $\begin{array}{l}\text { Severe atrophy } \\
(n=65)\end{array}$ & $\begin{array}{l}P \\
\text { value }\end{array}$ & Mild atrophy & Severe atrophy & $\begin{array}{l}P \\
\text { value }\end{array}$ \\
\hline $\begin{array}{l}\text { Initial dose of LEN } \\
(4 / 8 / 12 \mathrm{mg})\end{array}$ & $2 / 36 / 29$ & $6 / 41 / 18$ & 0.034 & & & \\
\hline $\begin{array}{l}\text { Dose down } \\
\text { (yes/no/unknown) }\end{array}$ & & & & $38 / 26 / 3$ & $26 / 38 / 1$ & 0.035 \\
\hline $\begin{array}{l}\text { Administration period of } \\
\text { LEN (months) }\end{array}$ & & & & $9.4(4.5-13.9)$ & $6.0(2.7-9.4)$ & 0.012 \\
\hline $\begin{array}{l}\text { Discontinued LEN } \\
\text { (yes/no) }\end{array}$ & & & & $48 / 19$ & $49 / 16$ & 0.63 \\
\hline $\begin{array}{l}\text { Reason for discontinuing } \\
\text { LEN (adverse } \\
\text { event/PD/other) ( } n=97)\end{array}$ & & & & $16 / 26 / 6$ & $23 / 18 / 8$ & 0.175 \\
\hline $\begin{array}{l}\text { Receiving other } \\
\text { treatments after } \\
\text { discontinuing LEN } \\
\text { (yes/no) }(n=97)\end{array}$ & & & & $37 / 11$ & $22 / 27$ & 0.001 \\
\hline $\begin{array}{l}\text { The } 1 \text { st mRECIST } \\
\text { assessment } \\
\text { (CR/PR/SD/PD/unknown) }\end{array}$ & $0 / 26 / 17 / 20 / 4$ & $0 / 20 / 22 / 22 / 1$ & 0.388 & & & \\
\hline Death (yes/no) & & & & $22 / 45$ & $30 / 35$ & 0.119 \\
\hline $\mathrm{PMI}\left(\mathrm{cm}^{2} / \mathrm{m}^{2}\right)$ & $5.61(4.55-7.23)$ & $5.66(4.79-6.61)$ & 0.902 & $6.04(4.43-7.05)$ & $4.26(3.59-5.32)$ & $<0.001$ \\
\hline \multicolumn{7}{|c|}{$\begin{array}{l}\text { Values are presented as median (interquartile range). LEN: lenvatinib, BMI: body mass index, ALBI: albumin-bilirubin, mALBI: } \\
\text { modified albumin-bilirubin, TNM: tumor node metastasis, HCC: hepatocellular carcinoma, LCL-C: low density lipoprotein cholesterol, } \\
\text { AFP: alpha fetoprotein, PIVKA-II: protein induced by Vitamin K absence or antagonists-II, PD: progressive disease, mRECIST: } \\
\text { Modified Response Evaluation Criteria in Solid Tumors, CR: complete response, PR: partial response, SD: stable disease, PMI: psoas } \\
\text { muscle index. }\end{array}$} \\
\hline \multicolumn{7}{|c|}{ Severe atrophy group: $\Delta \mathrm{PMI} / \mathrm{m}$ rate $\geq 1 \%$, Mild atrophy group: $\Delta \mathrm{PMI} / \mathrm{m}$ rate $<1 \%$. } \\
\hline \multicolumn{7}{|c|}{ ( $\triangle \mathrm{PMI} / \mathrm{m}$ rate: rate of change in $\mathrm{PMI}$ per month during administration of $\mathrm{LEN}$ ) } \\
\hline † Calculated using the ava & ole data. & & & & & \\
\hline
\end{tabular}

The LEN dose was reduced in fewer patients in the severe atrophy group compared with the mild atrophy group $(P=0.035)$. Fewer patients in the severe atrophy group received other treatments after discontinuing LEN compared with those in the mild atrophy group $(P=0.001)$. There was no significant difference in other HCC-related factors such as TNM stage and tumor markers between the groups.

\section{Adverse events}

An adverse event (AEs), regardless of grade, was experienced by 125 patients (94.7\%), and severe AEs (grade 3 or worse) occurred in 37 patients (28.9\%). There was no significant difference in any AEs, regardless of grade, between the low and normal PMI groups (Table S1). There was a significant difference in severe hypertension between the severe and mild atrophy groups (Table S2). There was no significant difference in the rate of severe AEs between the low and normal PMI groups $(29.0 \% \mathrm{vs.} 28.9 \%, P=0.978)$ or between the severe and mild muscle atrophy groups $(23.1 \%$ vs. $32.8 \%, P=0.175)$. There was no significant difference in BMI between patients with and without severe AEs $(P=0.918)$.

There was no difference in PMI-Pre or $\triangle \mathrm{PMI} / \mathrm{m}$ rate between patients who discontinued due to adverse events or progressive disease $(P$ $=0.54$ and $P=0.19$, respectively).

\section{Discussion}


In this study, we assessed change in SMV in patients with HCC who were treated with LEN. We found that SMV decreased during the administration period and that change in SMV mass during the administration period was significantly associated with poor prognosis in these patients.

Sarcopenia is associated with poor prognosis in patients with cirrhosis or HCC, independent of liver function reserve. ${ }^{9-14}$ Patients with $\mathrm{HCC}$ receive various treatments, along with assessment of both TNM stage and liver function reserve. In terms of treatment of HCC, sarcopenia is associated with a negative impact in HCC patients who undergo curative treatments such as hepatectomy and radiofrequency ablation. ${ }^{18}$ Most of these previous studies assessed SMV prior to initiation of treatment. Other non-curative treatment is often continued or repeated until response failure or intolerance to treatment occurs. The results of the present study suggest that because SMV can change during non-curative treatment (Fig. 1), the impact of SMV may be less at the start of non-curative treatment compared with its impact during curative treatment. Several studies have reported no significant association between sorafenib and hepatic intra-arterial therapy with SMV loss at baseline or OS, but identified change in SMV during these treatments as a significant prognostic factor for OS. ${ }^{15,16}$ In contrast, Imai et al reported an association of SMV loss with poor prognosis, both at baseline and during treatment. ${ }^{12}$ These findings imply that change in SMV during treatment may be a useful predictor of prognosis in patients with HCC receiving non-curative treatment, including LEN.

Several studies have identified change in SMV during treatment as an impact factor in HCC patients with treated with MTAs. ${ }^{12,15}$ However, these reports did not consider the impact of long-term change in SMV. In the present study, the median administration period of LEN was 7.7 months, and the impact of long-term change in SMV over at least 6 months was investigated in more than half of the enrolled patients.

In patients with HCC, SMV is affected by both liver function reserve and tumor-related factors in addition to age or sex. ${ }^{19}$ In fact, in the present study, median PMI-Pre, PMI-1st, and PMI-Post were 5.63,5.62, and $4.97 \mathrm{~cm}^{2} / \mathrm{m}^{2}$, respectively, and SMV had decreased significantly between Pre and Post. Furthermore, there was no significant difference in $\triangle \mathrm{PMI} / \mathrm{m}$ between the low and normal PMI groups. Similarly, Uchikawa et al reported a significant decrease in SMV during administration of MTAs, with or without muscle volume loss at baseline.$^{20}$ In the present study, liver function reserve was worse and ChE and albumin levels were lower in the severe atrophy group than in the mild atrophy group (Table 4). These results suggest that maintaining nutritional status and hepatic reserve prevents SMV loss. Takada et al reported that branch-chain amino acids (BCAAs) were useful for maintaining the serum albumin level, which helped to avoid early discontinuance of sorafenib therapy. ${ }^{21}$ Another study reported that in HCC patients treated with LEN, there was significant correlation between the plasma acyl carnitine-to-free carnitine ratio and change in the Brief Fatigue Inventory score, and mentioned that LEN affected carnitine insufficiency and fatigue. ${ }^{22}$ Accordingly, we should consider the early introduction of nutrition therapy, including BCAAs and levocarnitine, in HCC patients treated with MTAs.

In this study, non-mALBI 1 or $2 a$ at the start of LEN was a significant risk factor for OS (Table 3). Furthermore, the median ALBI score at baseline was -2.48 (mALBI grade $2 a$ ) in patients with mild atrophy and -2.31 (mALBI grade $2 b$ ) in those with severe atrophy (Table 4 ). Hiraoka et al reported that mALBI grade 1 or 2 a were better prognostic factors in LEN treatment ${ }^{23}$, and similar results were obtained in this study. The above findings suggest that a decrease in hepatic reserve leads to a rapid decrease in SMV, leading to a worsening of prognosis.

In the present patients with hypertension, severe AEs (grade 3 or worse) occurred more frequently in those in the mild than those in the severe atrophy group (Table S2), despite no significant difference in any grade of hypertension between the two groups. It is possible that blood pressure control was originally worse in more of the patients in the mild atrophy group compared with the severe atrophy group. There was no significant difference in any of the other AEs that affect patients' nutritional status such as diarrhea, anorexia, or body weight loss between either of the low and normal PMI groups or the severe and mild atrophy groups. Uojima et al have reported low SMV as a risk factor associated with severe AEs in patients treated with LEN, and that SMV was more important than body weight in those patients. ${ }^{14}$ In the present study, the initial dose of LEN was reduced as appropriate at the discretion of each researcher, and accordingly, AEs were unlikely to occur. However, doses of LEN were less reduced in patients in the severe atrophy group compared with those in the mild atrophy group. This result suggests that continued administration of excessive amounts of LEN may result in decreased hepatic reserve and SMV. A reduction in the dose of LEN should be considered in treatment of patients with SMV loss, even if AEs are mild. Furthermore, Hiraoka et al reported that the AE of appetite loss was more frequent in patients with lower BMI when a set dose was used and suggested that there might be a risk of LEN overdose in these patients even with a set dose. ${ }^{24}$ In the present study, lower BMI was not a risk factor for OS (Table 3) and there was no significant difference in BMI in terms of the severity of AEs. However,

Page $12 / 18$ 
BMI was lower in the present patients with severe atrophy than in those with mild atrophy (Table 4). Furthermore, the Pre values of ChE were lower in the severe atrophy group than in the mild atrophy group. These results suggest that patients with low BMI or poor nutritional status may be at risk of overdose, even with standard doses of LEN.

In the present study, we calculated SMV according to the simple method specified in the JSH guidelines for sarcopenia in liver disease. ${ }^{17}$ The JSH guidelines define SMI as the cross-sectional area of skeletal muscle at the level of L3 divided by height squared on CT. ${ }^{17}$ However, there is a demand for a simple screening method for sarcopenia that is more suitable for use in clinical practice, such as that described in the present study. In recent years, various researchers have proposed screening methods for sarcopenia using arm circumference, calf circumference, and body mass index. ${ }^{25-27}$ These simple methods are useful for evaluating changes in SMV during the treatment period for HCC and CLD.

The present study had several limitations in addition to its retrospective design and small sample size. First, as markers of muscular strength such as hand grip strength and walking speed were not evaluated, a diagnosis of sarcopenia was impossible according to the JSH guidelines. Second, we measured PMI by manual tracing on the CT images, which could have introduced errors due to inconsistency among the researchers. Third, we evaluated nutritional status only with respect to albumin, sugar metabolism, lipid metabolism and $\mathrm{ChE}$. Sano et al have reported a significant association of BCAA levels with sarcopenia, and a significant correlation of $\Delta$ leucine with $\triangle S M I(R=0.256, \mathrm{P}<0.001) .{ }^{28}$ Okubo et al have reported that sarcopenia was diagnosed significantly more frequently in patients with vitamin D deficiency compared with those without vitamin D deficiency. ${ }^{29}$ In addition, we did not evaluate liver fibrosis by hyaluronic acid, Mac-2 binding protein glycosylated isomer, or elastography (e.g., transient elastography, magnetic resonance). To prevent sarcopenia and thus improve prognosis in HCC patients treated with LEN, prospective large-scale and interventional studies are needed to overcome the above-mentioned limitations.

In conclusion, progressive loss of SMV was associated with OS in patients with HCC during administration of LEN. Prevention of SMV loss, including nutrition therapy, is essential for improving prognosis in HCC patients treated with LEN.

\section{Methods}

\section{Patients}

We retrospectively analyzed 171 patients with advanced u-HCC who were treated with LEN between April 2018 and July 2020 at one of the following hospitals in Japan: Fukushima Medical University Hospital, Iwate Medical University Hospital, Tohoku University Hospital, Yamagata University Hospital, Akita University Hospital, Hirosaki University Hospital, and National Hospital Organization Sendai Medical Center. Patients who had received other treatments such as surgery, local therapy, hepatic intra-arterial therapy, radiation therapy, and chemotherapy including sorafenib before the start of LEN administration were included in the study. Excluded were 39 patients in whom a large amount of data were missing at the start of LEN, or in whom SMI could not be measured because magnetic resonance imaging (MRI) was performed instead of CT. In total, 132 patients were enrolled in the study. All enrolled patients had been treated only with LEN from the start of LEN administration (Pre) to the end of LEN administration (Post). If a patient's liver function was good enough to permit administration of other treatments (MTAs, hepatic arterial infusion chemotherapy, or radiation therapy) at Post, these treatments were added to the regimen. The study protocol conformed to the ethics guidelines of the Declaration of Helsinki. The study protocol was reviewed and opt-out consent was approved by the Ethics Committee of Fukushima Medical University (No. 2019233). The need to obtain informed consent from the participants was waived by the Ethics Committee of Fukushima Medical University due to the retrospective nature of the study.

\section{Evaluation of HCC}

The diagnosis of HCC was performed by CT or MRI. TNM stage was determined according to the Japanese criteria. ${ }^{30}$ The size and number of HCCs were evaluated using the Up-to-7 criteria. ${ }^{31}$ Therapeutic effect of LEN was assessed by CT at 1-3 months after the start of administration of LEN according to the Modified Response Evaluation Criteria in Solid Tumors (mRECIST). ${ }^{32}$ Afterwards, patients were followed up by CT every 3 months.

\section{Assessment of liver function reserve}

Liver function reserve was evaluated by the Child-Pugh classification ${ }^{33}$, ALBI score, and mALBI grade. $^{34,35}$

\section{Treatment}

Page $13 / 18$ 
All patients received $4 \mathrm{mg} / 8 \mathrm{mg} / 12 \mathrm{mg}$ LEN once daily based on their body weight and liver function reserve. The initial dose of LEN was reduced as appropriate, at the discretion of each researcher. When any grade 3 or worse severe AEs or any unacceptable grade 2 AEs occurred, the dose of LEN was reduced or withdrawn until symptoms subsided, according to the guidelines provided by the manufacturer. AEs were assessed according to the National Cancer Institute Common Terminology Criteria for Adverse Events, version 4.0. The administration period (months) was defined as the period between the times of the start and end of LEN administration. If any of the following criteria were met, the administration of LEN was discontinued: (i) development of any unacceptable or serious AEs, (ii) progressive disease (PD) of the tumor as defined by mRECIST, (iii) worsening liver function reserve, or (iv) request by the patient to discontinue LEN.

\section{Evaluation of change in skeletal muscle volume and characteristics}

The JSH guidelines for sarcopenia in liver disease recommend evaluation by SMI as the total muscle volume of four limbs measured by bioelectrical impedance analysis (BIA) divided by height squared. ${ }^{17}$ A simpler method for determining SMI is by PMI, calculated as the left-right sum of the vertical $\times$ horizontal diameter of the psoas muscle at the level of L3 on CT, divided by height squared ${ }^{16}$, which is indirectly correlated with SMI obtained using BIA. ${ }^{17}$ Thus, we used PMI in the present study. We measured PMI on CT images obtained at the start of LEN administration (PMI-Pre), at the time of the first judgement of therapeutic effect (PMI-1st), and at the end of LEN administration (PMI-Post). Patients were classified into two groups based on the cut-off values specified by JSH guidelines for sarcopenia in liver disease: low PMI group (PMI $<6.0 \mathrm{~cm}^{2} / \mathrm{m}^{2}$ for men and $<3.4 \mathrm{~cm}^{2} / \mathrm{m}^{2}$ for women) and normal PMI group (PMI $\geq 6.0$ $\mathrm{cm}^{2} / \mathrm{m}^{2}$ for men and $\geq 3.4 \mathrm{~cm}^{2} / \mathrm{m}^{2}$ for women). ${ }^{17}$

As an index of progressive muscle atrophy, we calculated $\Delta \mathrm{PMI} / \mathrm{m}$ with the following formula: (PMI-Pre - PMI-Post)/administration period. ${ }^{16}$ The rate of decrease in PMI per month $\left(\triangle \mathrm{PMI} / \mathrm{m}\right.$ rate; $\left.\Delta \mathrm{PMI}{ }^{*} 100 / \mathrm{PMI}-\mathrm{Pre}\right)$ was also calculated. Patients were classified into two groups (severe and mild atrophy groups) according to $\Delta \mathrm{PMI} / \mathrm{m}$ of $\geq 1 \%$ or $<1 \%$, respectively.

Characteristics including age, liver function reserve, stage, laboratory findings and prognosis including OS (months), MST, and survival rate were evaluated.

\section{Statistical analysis}

Continuous variables are expressed as the median and interquartile range (IQR). Statistical analyses were performed using the $\chi^{2}$ test, Fisher's exact test, the Mann-Whitney U test, Kruskal-Wallis rank sum test, or Wilcoxon signed rank test, as appropriate. OS was evaluated by Kaplan-Meier analysis, using the log-rank test. Cox proportional hazard analysis was used to determine the risk factors for OS. In the case of missing values, statistical analysis was performed with the available data. All P-values were two-tailed, and Pvalues $<0.05$ were considered statistically significant. Statistical analyses were performed using Easy R (http://www.jichi.ac.jp/saitamasct/SaitamaHP. .les/statmed.html). ${ }^{36}$

\section{Declarations}

\section{Acknowledgments}

We would like to thank all participants for their cooperation in data collection and for providing

information during the study period.

\section{Author Contributions}

M.F. conceived and designed the study; K.A., H.K., T.O., M.N., K.O, T.K., W.S., T. E., N. T.

and H. N. collected the data; M.F. analyzed the data; M.F., K.A. and H.O. drafted the

manuscript. All authors reviewed the final manuscript.

\section{Data Availability Statement}

All data generated or analyzed during this study are included in this article. Further inquiries can

be directed to the corresponding author.

Page $14 / 18$ 


\section{References}

1. Parki, D. M., Bray, F., Ferlay, J. \& Pisani, P. Estimating the world cancer burden: GLOBOCAN 2000. Int J Cancer. 94, 153-56 (2001).

2. Kokudo, N. et al. Clinical practice guidelines for hepatocellular carcinoma: The Japan Society of Hepatology 2017 (4th JSH-HCC guidelines) 2019 update. Hepatol Res. 49, 1109-13 (2019).

3. Llovet, J.M. et al. Sorafenibb in advanced hepatocellular carcinoma. N Engl J Med. 359, 378-90 (2008).

4. Bruix, J.et al. Regorafenib for patients with hepatocellular carcinoma who progressed on sorafenib treatment (RESORCE): a randomised, double-blind, placebo-controlled, phase 3 trial. Lancet. 389, 56-66 (2017).

5. Kudo, M. et al. Lenvatinib versus sorafenib in first-line treatment of patients with unresectable hepatocellular carcinoma: a randomised phase 3 non-inferiority trial. Lancet. 391, 1163-73 (2018).

6. Zhu, A.X., Finn, R.S., Galle, P.R., Llovet, J.M. \& Kudo, M. Ramucirumab in advanced hepatocellular carcinoma in REACH-2: the true value of alpha-fetoprotein. Lancet Oncol. 20, e191 (2019).

7. Finn, R.S. et al. Atezolizumab plus bevacizumab in unresectable hepatocellular carcinoma. N Engl J Med. 382, 1894-905 (2020).

8. Kudo, M. Recent advances in systemic therapy for hepatocellular carcinoma in an Aging Society: 2020 update. Liver Cancer. 9 , 640-62 (2020).

9. Hanai, T.et al. Rapid skeletal muscle wasting predicts worse survival in patients with liver cirrhosis. Hepatol Res.46, $743-51$ (2016).

10. Hiraoka, A. et al. Muscle atrophy as pre-sarcopenia in Japanese patients with chronic liver disease: computed tomography is useful for evaluation. J Gastroenterol. 50, 1206-13 (2015).

11. Hiraoka, A. et al. Muscle volume loss as a prognostic marker in hepatocellular carcinoma patients treated with sorafenib. Hepatol Res. 47, 558-65 (2017).

12. Imai, K. et al. Rapid depletions of subcutaneous fat mass and skeletal muscle mass predict worse survival in patients with hepatocellular carcinoma treated with sorafenib. Cancer. 11, 1206, (2019).

13. Hiraoka, A. et al. Clinical importance of muscle volume in lenvatinib treatment for hepatocellular carcinoma: Analysis adjusted with inverse probability weighting. J Gastroenterol Hepatol. doi: 10.1111/jgh.15336 (2020).

14. Uojima, H. et al. Skeletal muscle mass influences tolerability and prognosis in hepatocellular carcinoma patients treated with lenvatinib. Liver Cancer. 9, 193-206 (2020).

15. Yamashima, M.et al. Significance of psoas muscle thickness as an indicator of muscle atrophy in patients with hepatocellular carcinoma treated with sorafenib. Mol Clin Oncol. 7, 449-53 (2017).

16. Fujita, M. et al. Skeletal muscle volume loss during transarterial chemoembolization predicts poor prognosis in patients with hepatocellular carcinoma. Hepatol Res. 49, 778-86 (2019).

17. Nishikawa, H. et al. Japan Society of Hepatology guidelines for sarcopenia in liver disease (1st edition): Recommendation from the working group for creation of sarcopenia assessment criteria. Hepatol Res. 46, 951-63 (2016).

18. Chang, K.V. et al. Association between loss of skeletal muscle mass and mortality and tumor recurrence in hepatocellular carcinoma: a systematic review and meta-analysis. Liver Cancer. 7, 90-103 (2018).

19. Imai, K. et al. Sarcopenia impairs prognosis of patients with hepatocellular carcinoma: The role of liver functional reserve and tumor-related factors in loss of skeletal muscle volume. Nutrients.doi: 10.3390/nu9101054 (2017).

20. Uchikawa, S. et al. Skeletal muscle loss during tyrosine kinase inhibitor treatment for advanced hepatocellular carcinoma patients. Liver Cancer. 9, 148-55 (2020).

21. Takeda, H. et al. Effect of treatment with branched-chain amino acids during sorafenib therapy for unresectable hepatocellular carcinoma. Hepatol Res. 44, 302-12 (2014).

22. Okubo, H. et al. Carnitine insufficiency is associated with fatigue during lenvatinib treatment in patients with hepatocellular carcinoma. PLoS One. 15, e0229772 (2020).

23. Hiraoka, A. et al. Post-progression treatment eligibility of unresectable hepatocellular carcinoma patients treated with lenvatinib. Liver Cancer. 9, 73-83 (2020).

24. Hiraoka, A. et al. Prognostic factor of lenvatinib for unresectable hepatocellular carcinoma in real-world conditions-Multicenter analysis. Cancer Med. 8, 3719-28 (2019).

25. Endo, K., Sato, T., Kakisaka, K. \& Takikawa, Y. Calf and arm circumference as simple markers for screening sarcopenia in patients with chronic liver disease. Hepatol Res. 51, 176-89 (2021).

Page 15/18 
26. Ichikawa, T. et al. Calculated body muscle mass as a useful screening marker for low skeletal muscle mass and sarcopenia in chronic liver disease. Hepatol Res. 50, 704-14 (2020).

27. Nishikawa, H. et al. Clinical impact of the finger-circle test in patients with liver diseases. Hepatol Res. 51, 603-13 (2021).

28. Sano, A. et al. Plasma free amino acids are associated with sarcopenia in the course of hepatocellular carcinoma recurrence. Nutrition. doi: 10.1016/j.nut.2020.111007 (2020).

29. Okubo, T. et al. Relationship between serum vitamin D level and sarcopenia in chronic liver disease. Hepatol Res. 50, 588-97 (2020).

30. The Liver Cancer Study Group of Japan. The General Rules for the Clinical and Pathological Study of Primary Liver Cancer, 6th English edition. 26 (Tokyo: Kanehara \& CO., Ltd 2020).

31. Mazzaferro, V. et al. Predicting survival after liver transplantation in patients with hepatocellular carcinoma beyond the Milan criteria: a retrospective, exploratory analysis. The Lancet Oncol. 10, 35-43 (2009).

32. Lencioni, R. \& Llovet, J.M. Modified RECIST (mRECIST) assessment for hepatocellular carcinoma. Semin Liver Dis. 30, 52-60 (2010).

33. Pugh, R.N., Murray-Lyon, I.M., Dawson,J.L., Pietroni, M.C. \& Williams, R. Transection of the oesophagus for bleeding oesophageal varices. Br J Surg. 60, 646-9 (1973).

34. Hiraoka, A. et al. Validation and potential of albumin-bilirubin grade and prognostication in a nationwide survey of 46,681 hepatocellular carcinoma patients in Japan: the need for a more detailed evaluation of hepatic function. Liver Cancer. 6, 325-336 (2017).

35. Hiraoka, A. et al. Validation of modified ALBI grade for more detailed assessment of hepatic function in hepatocellular carcinoma patients: a multicenter analysis. Liver Cancer 8, 121-9 (2019).

36. Kanda, Y. Investigation of the freely available easy-to-use software 'EZR' for medical statistics. Bone Marrow Transplant. 48, 452-8 (2013).

\section{Figures}




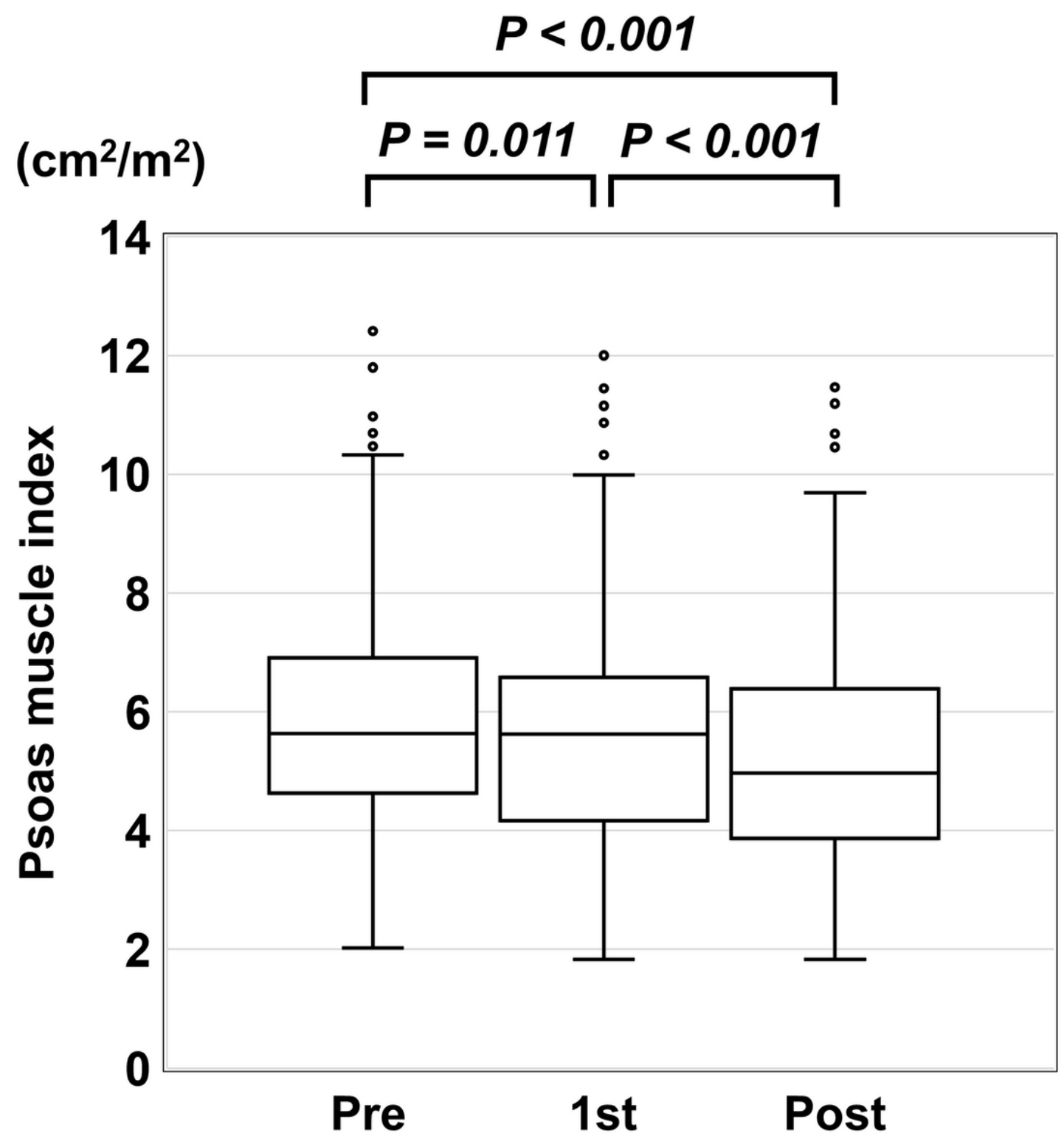

Figure 1

Transition of change in psoas muscle index (PMI) before (pre), during $\left(1^{\text {st }}\right)$, and after (post) administration of lenvatinib.

There was a significant decrease in PMI at each successive measurement during the study period. 
(A)

$(\%)$

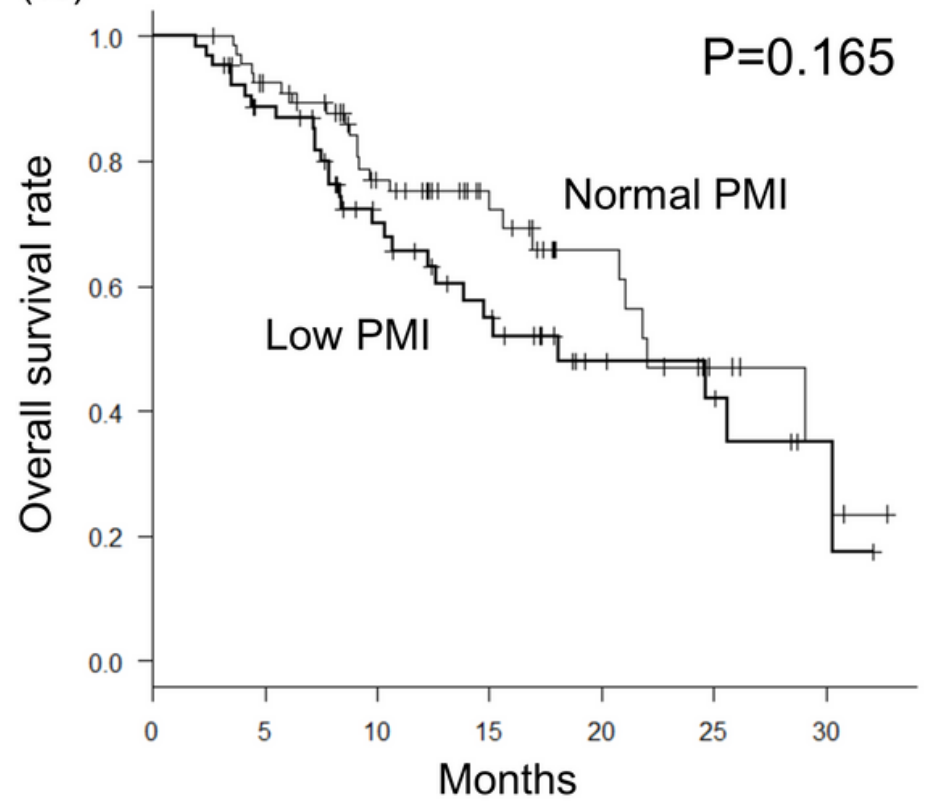

(B)

$(\%)$

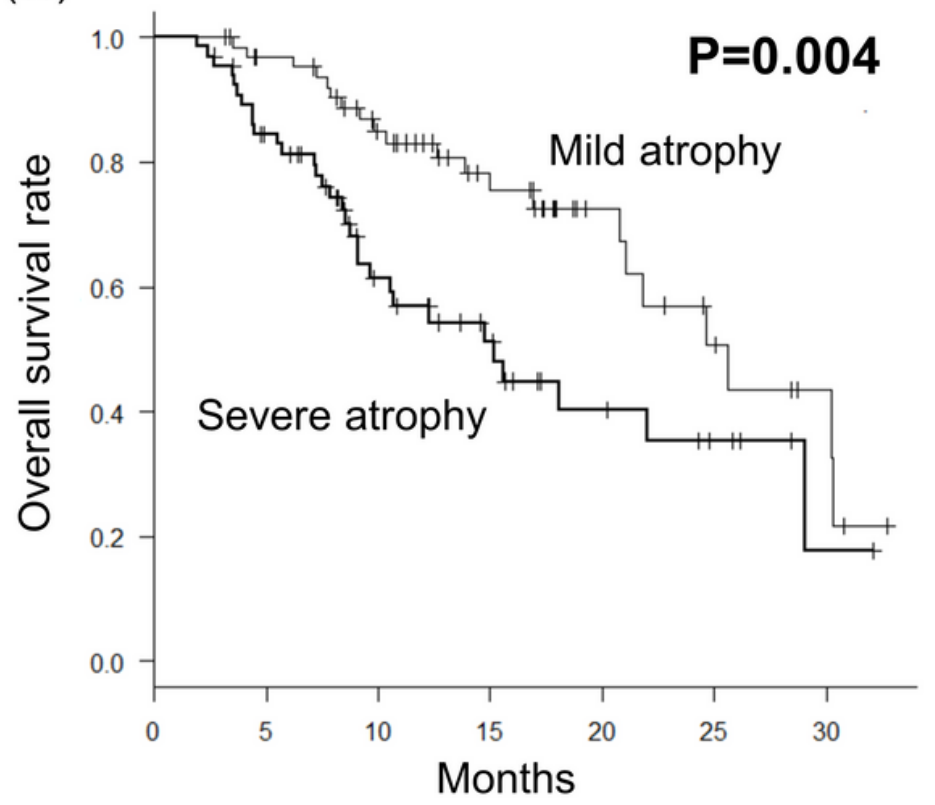

Figure 2

Kaplan-Meier curves for overall survival in the low and normal PMI groups (A) and in the severe and mild atrophy groups (B).

\section{Supplementary Files}

This is a list of supplementary files associated with this preprint. Click to download.

- TableSupplement.pdf 